\title{
CALIDAD DE VIDA DEL PACIENTE CON DIABETES MELLITUS TIPO 2 Y CONTROL PREVENTIVO DE LA ENFERMEDAD EN EL HOSPITAL REGIONAL DE ICA, 2010
}

\section{Quality of life of patients with Type 2 Diabetes Mellitus prevention and control of the disease. Hospital Regional de Ica. 2010.}

Alejandrina Bertha Pebes Mendoza ${ }^{1, a, b}$

\footnotetext{
${ }^{1}$ Facultad de Enfermería. Universidad Nacional San Luis Gonzaga de Ica. Ica, Perú

${ }^{a}$ Licenciada en Enfermería' ${ }^{b}$ Doctor en Salud Publica.
}

\section{RESUMEN}

Se sabe relativamente poco acerca de cómo la diabetes afecta la calidad de vida de las personas, debido a que este concepto difiere en su definición y en la forma de medirlo.

Objetivo: Determinar la relación que existe entre el Control Preventivo de la Enfermedad y la Calidad de Vida de pacientes con Diabetes Mellitus tipo 2. Material y métodos: El diseño utilizado es descriptivo correlacional prospectivo, Con una población de todos los pacientes que se han atendido en el Hospital Regional de Ica, con Diabetes Mellitus tipo 2, se consideró muestra de 60 pacientes, la técnica utilizada fue análisis documental, encuestas y cuestionarios. Resultados: Con respecto a los pacientes con Diabetes Mellitus tipo 2 se encontró que el $22 \%$ estuvo satisfecho, $78 \%$ insatisfecho con su calidad de vida, $63 \%$ tuvieron control de su enfermedad, $22 \%$ lo hicieron algunas veces, $15 \%$ de ellos nunca, $47 \%$ de pacientes nunca tuvieron control preventivo de la enfermedad, $38,67 \%$ lo hicieron a veces y $14,33 \%$ lo hizo siempre, Conclusiones: Por consiguiente concluimos que el control preventivo de la diabetes mellitus tipo 2 está relacionada con la calidad de vida de los pacientes, es decir si existe mejor control y tratamiento de la enfermedad, el autocuidado con cumplimiento del cuidado de la vista, los pies, cuidado dietético y ejercicios mejorará la calidad de vida de los pacientes que cursan esta enfermedad.

Palabras claves: Diabetes Mellitus, calidad de vida, prevención.

\section{SUMMARY}

Relatively little is known about how diabetes affects the quality of life of people, because this concept differs in its definition and how to measure it. Objective: To determine the relationship between the Preventive Disease Control and Quality of Life of Patients with Type 2 Diabetes Mellitus. Material and Methods: The design used is descriptive prospective correlational With a population of all patients who have been treated at the Regional Hospital of Ica, with type 2 diabetes was considered sample of 60 patients, the technique used was documentary analysis, surveys and questionnaires. Results: With regard to patients with type 2 diabetes found that $22 \%$ were satisfied, $78 \%$ dissatisfied with their quality of life, $63 \%$ had control of their disease, $22 \%$ did so sometimes, $15 \%$ never , $47 \%$ of patients never had preventive disease control, $38,67 \%$ did so sometimes and $14,33 \%$ always did, Conclusions: We therefore conclude that the preventive management of diabetes we mellitus type 2 is related to the quality of life of patients, ie if there is better control of the disease and treatment, compliance with self-care eye care, feet care dietary exercises and improve the quality of life of patients enrolled in this disease.

Keywords: Diabetes mellitus, quality of life, prevention. 


\section{INTRODUCCION}

Según la OMS, define la Diabetes Mellitus como un estado de hiperglucemia crónica producidos por diversos factores: genéticos, alimenticios, etc. La hormona insulina secretada por las células Beta del Páncreas el principal regulador de la concentración de azúcar en la sangre. Cuando esta hormona no se produce eficientemente la concentración del azúcar se incrementa (1). Calidad de vida como la percepción que un individuo tiene de su lugar en la existencia, en el contexto de la cultura y del sistema de valores en los que vive y en relación con sus objetivos, sus expectativas, sus normas, sus inquietudes, es un concepto muy amplio que está influido de modo complejo por la salud física del sujeto, su estado psicológico, su nivel de independencia, sus relaciones sociales, así como su relación con los elementos esenciales de su entorno (2). La calidad de la vida significa calidad de existencia de los seres humanos concretos y reales, y en las situaciones específicas en que existen y se esfuerzan por existir. Otra forma de definir el concepto calidad de vida, es el de comprenderlo en la satisfacción de las necesidades básicas (3).

Las estadísticas señalan que el $3-4 \%$ de la población es diabética. La edad de máxima de aparición es alrededor de los 60 años, siendo en la tercera edad el porcentaje de 5$10 \%$. En niños la frecuencia es de 1 por cada 500-1000 niños. En diversos estudios epidemiológicos se ha podido comprobar que aproximadamente entre un $40 \%$ y $60 \%$ de los diabéticos padecen hipertensión, la hipertensión suele diagnosticarse años después del comienzo de la diabetes (4).

La Diabetes es una de las enfermedades crónicas no transmisibles de mayor prevalencia. Según la OMS, "todos los años mueren en el mundo 3,2 millones de personas por complicaciones directamente relacionadas con la diabetes, como retinopatía, neuropatía y nefropatía diabética. En América Latina la situación es grave ya que hay 13,3 millones de enfermos y se calcula que habrá 33 millones de enfermos en el 2030". Actualmente el Perú se encuentra entre los cinco países, con mayor número de casos, siendo antecedido por Brasil, México, Argentina y Colombia. En nuestra región esta situación se ve de manera concreta en el Hospital Regional de Ica, donde según la oficina de estadística e informática durante el año 2008 se han registrado 400 casos de diabetes y 58 hospitalizaciones, en el 2009, se registraron 374 casos y 61 hospitalizaciones. Dentro del auto cuidado que deben realizar los pacientes diabéticos se encuentra el cumplimiento con el cuidado farmacológico, cuidado de la vista, cuidado dietético y ejercicios. Como consecuencia del incumplimiento de estos cuidados se presentan complicaciones que llevan al paciente a hospitalizarse lo que genera gasto económico al hospital e implicancias en el aspecto biopsicosocial - espiritual del paciente (5).

Esta enfermedad requiere del servicio del personal de enfermería, dentro de sus acciones preventivo promocionales cumple un rol muy importante en el cuidado del paciente diabético ya que realiza una serie de acciones, dirigidas a los diferentes factores que favorecen o limitan el auto cuidado del paciente en la prevención de complicaciones promoviendo estilos de vida saludables, mejorando su calidad de vida, contribuyendo así a disminuir las altas tasas de morbimortalidad de la diabetes (6).

El presente trabajo de investigación tuvo como objetivo Determinar la relación que existe entre el control preventivo de la enfermedad y la calidad de vida de pacientes con Diabetes Mellitus Tipo 2. 


\section{MATERIAL Y METODOS}

El presente trabajo de investigación es Descriptivo, prospectivo, Correlacional, con una población compuesta por todos los pacientes con Diabetes Mellitus Tipo 2 que acuden al Hospital Regional de Ica. La muestra fue de tipo probabilístico, fórmula utilizada para el caso de poblaciones infinitas, con un nivel de $95 \%$ de confianza $n=60$. Antes de realizar la prueba de hipótesis se determinó el tipo de instrumento que se utilizó para la recolección de datos, a fin de establecer si éstos obedecen a la estadística planteada. Para determinar el grupo de confiabilidad se estimó el coeficiente de confiabilidad mediante el coeficiente de correlación de Pearson y la ecuación de Spearman - Brown mediante el método de mitades divididas. Para determinar la calidad de vida de los pacientes con Diabetes Mellitus tipo 2 y su grado de satisfacción. Tomamos los 7 primeros promedios más frecuentes y los 7 últimos, existiendo alta confiabilidad. Para el control preventivo de la enfermedad y su grado de atención o escala. Consideramos los 5 primeros y 5 últimos, Existiendo una significativa confiabilidad. Para el control preventivo y su tratamiento consideramos los pares e impares, Existiendo alta confiabilidad.

Análisis estadístico.- Para la Prueba de hipótesis de medias poblacionales, se utilizó un análisis no paramétrico de chi- cuadrado y análisis de correlación a fin de contrastar la hipótesis estadística planteada y verificar su validación.

\section{RESULTADOS}

Con una población de todos los pacientes que se han atendido en el Hospital Regional con Diabetes Mellitus tipo 2 se tomó una muestra de 60 pacientes encontrándose que, el $42 \%$ es de sexo masculino y el $58 \%$ de sexo femenino, de estos, según su ocupación el $20 \%$ son obreros, el $38 \%$ son empleados y el $42 \%$ realizan labores de hogar, de todos ellos el $55 \%$ son casados, el $41 \%$ tienen sus edades comprendidos entre 51 y 61 años.

Tabla 1. Datos generales de los pacientes con diabetes Mellitus

Tipo 2 Hospital Regional-2010

$\begin{array}{lcc}\text { Sexo } & \text { FI } & \% \\ \text { Femenino } & 35 & 58 \\ \text { Masculino } & 25 & 42 \\ \text { Total } & \mathbf{6 0} & \mathbf{1 0 0} \\ \text { Ocupación } & \mathbf{f i} & \% \\ \text { Obrero } & 12 & 20 \\ \text { Empleado } & 23 & 38 \\ \text { Labores De Hogar } & 25 & 42 \\ \text { Total } & \mathbf{6 0} & \mathbf{1 0 0} \\ \text { Estado Civil } & \mathbf{f i} & \% \\ \text { Soltero } & 9 & 15 \\ \text { Casado } & 33 & 55 \\ \text { Viudo } & 12 & 20 \\ \text { Divorciado } & 3 & 5 \\ \text { Conviviente } & 2 & 3 \\ \text { Separado } & 1 & 2 \\ \text { Total } & \mathbf{6 0} & \mathbf{1 0 0} \\ \text { Edad En Años } & \mathbf{f i} & \% \\ 18-28 & 1 & 2 \\ 29-39 & 1 & 2 \\ 40-50 & 8 & 13 \\ 51-61 & 25 & 41 \\ 62-72 & 13 & 22 \\ 73-83 & 10 & 17 \\ 84-94 & 2 & 3 \\ \text { TOTAL } & \mathbf{6 0} & \mathbf{1 0 0} \\ & & \end{array}$


La calidad de vida de los pacientes con Diabetes Mellitus 2 se ha medido de acuerdo a la satisfacción que tienen con respecto a la enfermedad de tal manera que se ha encontrado que el $78 \%$ están insatisfechos, el $22 \%$ están satisfechos con su calidad de vida, así mismo, el $15 \%$ de estos pacientes nunca tuvieron control de la enfermedad, el $22 \%$ algunas veces y el $63 \%$ casi siempre, con respecto al control preventivo de la enfermedad, $47 \%$ nunca lo hicieron, $38,67 \%$ lo hicieron a veces y sólo el $14,33 \%$ lo hacen siempre.

Tabla 2. Calidad de Vida, Control de enfermedad y Control Preventivo de Pacientes con Diabetes Mellitus Tipo 2

\begin{tabular}{llr} 
Calidad de vida & $\mathbf{f i}$ & $\%$ \\
Satisfecho & 101 & 22 \\
Insatisfecho & 362 & 78 \\
\hline Total & 463 & 100 \\
\hline Control de la enfermedad & & \\
Nunca & 84 & 15 \\
Algunas veces & 121 & 22 \\
Casi siempre & 346 & 63 \\
\hline Total & 551 & 100 \\
\hline Control Preventivo de la Enfermedad & \multicolumn{2}{|}{} \\
Nunca & 28 & 47 \\
A Veces & 23 & 38,67 \\
Siempre & 9 & 14,33 \\
$\quad$ Total & 60 & 100.00
\end{tabular}

Con respecto al control preventivo de la enfermedad se ha tomado en cuenta aspectos importantes de autocuidado que el paciente debe cumplir para una buena calidad de vida, sin embargo los porcentajes encontrados en el presente trabajo fueron que el $10 \%$ nunca consideraron alimentarse con una dieta adecuada, 63,33\% consideraron a veces y $26,67 \%$ si consideró siempre alimentarse de forma adecuada, el 58,33\% de los pacientes nunca realizan ejercicios, el $31,67 \%$ a veces y el $10 \%$ de estos si hacen ejercicios siempre, el $83,33 \%$ nunca tuvieron cuidados con los pies, el $10 \%$ a veces y el $6,67 \%$ siempre tienen cuidado con los pies, finalmente el $33,33 \%$ de los pacientes nunca toman medicamentos, el $45 \%$ lo hacen a veces y el $21,67 \%$ lo hacen siempre.

Tabla 3. Control Preventivo Y Su Grado De Tratamiento De Pacientes Con Diabetes Mellitus 2

\begin{tabular}{|c|c|c|c|c|c|c|c|c|}
\hline \multirow{3}{*}{ CONTROL } & \multicolumn{6}{|c|}{ TRATAMIENTO } & \multirow{2}{*}{\multicolumn{2}{|c|}{ TOTAL }} \\
\hline & \multicolumn{2}{|c|}{ NUNCA } & \multicolumn{2}{|c|}{ A VECES } & \multicolumn{2}{|c|}{ SIEMPRE } & & \\
\hline & fi & $\%$ & fi & $\%$ & fi & $\%$ & $\mathrm{fi}$ & $\%$ \\
\hline Dietas & 6 & 10 & 38 & 63,33 & 16 & 26.67 & 60 & 100 \\
\hline Ejercicios & 35 & 58,33 & 19 & 31,67 & 6 & 10 & 60 & 100 \\
\hline Exámenes de azúcar & 30 & 50 & 26 & 43,33 & 4 & 6,67 & 60 & 100 \\
\hline Cuidado de los pies & 50 & 83,33 & 6 & 10 & 4 & 6,67 & 60 & 100 \\
\hline Medicamentos & 20 & 33,33 & 27 & 45 & 13 & 21,67 & 60 & 100 \\
\hline
\end{tabular}




\section{DISCUSIÓN}

El presente estudio se realizó en el Hospital Regional de Ica considerando la Calidad de vida de los pacientes con Diabetes Mellitus tipo 2, control de la enfermedad y el control preventivo de la misma Con una población de todos los pacientes que se han atendido en el Hospital, encontrándose que el $22 \%$ de los pacientes se encuentran satisfechos y el $78 \%$ insatisfechos con su calidad de vida, un alto porcentaje de insatisfacción en los pacientes debido a que estos no tienen tiempo para manejar su enfermedad, no tienen tiempo para su control y tratamiento. Con respecto al control de la enfermedad, los pacientes en estudio manifestaron que nunca hicieron control de su enfermedad en $15 \%$, algunas veces en $22 \%$ y casi siempre controlan su enfermedad en un $63 \%$ así mismo referente al control preventivo de la enfermedad se tiene un $47 \%$ no tienen control preventivo de su enfermedad, $38,67 \%$ a veces y $14,33 \%$ siempre. Estos hallazgos son semejantes a los resultados obtenidos por HAROL GARCíA BENAVIDES, Ana C. Portocarrero Curay, en su tesis Autocuidado de personas con Diabetes Mellitus tipo 2, del Hospital de Apoyo "José Alfredo Mendoza Olavarría" 2005. Concluye que en el apoyo familiar, la familia cumple un rol muy importante apoyando al paciente diabético en su cuidado personal y autocuidado a diferencia del resultado obtenido en mi trabajo que el 50\% de la familia de los pacientes estudiados se encuentran insatisfechos y solo el $23 \%$ se encuentran satisfechos de brindar apoyo a su familiar (7).

NODA MILLA Julio Roberto y Col. En su tesis Nivel de conocimiento sobre Diabetes Mellitus tipo 2 en pacientes hospitalizados. Concluyo que el nivel de conocimiento sobre la enfermedad fue adecuado en un $12,9 \%$, intermedio el $48,39 \%$ y el $38,71 \%$ fue inadecuado por lo tanto en nivel de conocimientos fue insuficiente. A diferencia del presente trabajo donde el $60 \%$ de pacientes se encuentran insatisfechos, el $23 \%$ satisfechos y un $10 \%$ completamente insatisfechos; solo un 7\% se encontró completamente satisfecho de los conocimientos sobre su enfermedad (8).

A. HERVAS, Y COL. En su tesis Impacto de la Diabetes Mellitus en la Calidad de Vida relacionada con la Salud 2008. Concluye que la Diabetes Mellitus se relaciona con una Peor percepción de Calidad de Vida relacionada con la salud; similar a la conclusión del presente trabajo donde los pacientes con Diabetes Mellitus Tipo 2, experimentan una Pobre Calidad de Vida recayendo fundamentalmente en las personas de mayor edad quienes son las más vulnerables (9).

\section{CONCLUSIONES}

Por consiguiente concluimos que el control preventivo de la diabetes mellitus tipo 2 está relacionada con su calidad de vida, es decir si existe mejor control de la enfermedad, la calidad de los pacientes mejorará en consecuencia que la calidad de vida depende de su tratamiento y control preventivo.

\section{Correspondencia:}

Correo Electrónico:

Bertha-pebes18@hotmail.com

\section{REFERENCIAS BIBLIOGRAFICAS}

1. Organización Mundial de la Salud (OMS). Diabetes mellitus tipo 1, 2; (Visitada 2308-2010, 11 am.) Disponible en: http://www.ferato.com/wiki/index. php/Diabetes Mellitus

2. Oblitas, L. Psicología de la salud y calidad de vida, segunda edición, Buenos Aires: THOMSON. 2006. (visitada 22 08 - 2010. 10 Am.) Disponible en: http://www.findeen.es/calidad de vida 0 ms concepto.html

3. Maldonado, C. Comparaciones interpersonales e interculturales de bienestar. En: Bioética y Calidad de Vida, colección BíOS Y ETHOS, V. 15, Bogotá, Colombia. D.C. Ediciones El Bosque. 2000. (visitada 03-05-2010, 5pm.). Disponible en: http://www.salud.com/ secciones/diabetes.asp?contenido $=1368$ 
4. Islas A. Diabetes mellitus. $2^{\circ}$ ed. México: 2010. McGraw-Hill Interamericana. 1999. (visitada el 03 - 05 - 2010. 4Pm.) Disponible en: http://www.who.int/topic s/diabetes mellitus/es/.

5. Organización Mundial de la Salud (OMS). La diabetes mellitus. (DM). (visitada el 23- 09- 2010, 10 Pm.). Disponible en: www.who.int/topics/diabetes mellitus/es

6. Bravo E. [Internet] Disponible en: htttp://webcache.googleusercontent.com/ search?q=cache:ALXPHSSPivsJ:www.en fermeriajw.cl/teoristas/DOROTHEA.ppt+o rem+autocuidado\&cd=3\&hl=es\&ct=clnk\& $\mathrm{gl}=$ pe. (visitada 22-09-2010,11 pm.)

7. Velia M. Calidad de vida del paciente con diabetes mellitus tipo 2, Nuevo LeonMéxico: 2005 "

8. García H. "Autocuidado de personas con Diabetes Mellitus del Hospital de Apouo "José Alfredo Mendoza Olavarria" Tumbes, Perú. Octubre 2004 - Enero 2005
9. Noda J. Conocimientos sobre su enfermedad en pacientes con diabetes mellitus tipo 2 que acuden a hospitales generales. Rev Med Hered v.19 n.2 Lima, Perú. 2008.

10. Hervás A. "Calidad de vida relacionada con la salud en pacientes con diabetes mellitus tipo 2" España; 2008.

Recibido: 18/02/14

Aprobado para Publicación: 22/05/14 